\title{
Spectral discontinuities and the vowel length effect
}

\author{
ANDREW J. LOTTO and KEITH R. KLUENDER \\ University of Wisconsin, Madison, Wisconsin \\ and \\ KERRY P. GREEN \\ University of Arizona, Theson, Arizona
}

\begin{abstract}
Perception of voicing for stop consonants in consonant-vowel syllables can be affected by the duration of the following vowel so that longer vowels lead to more "voiced" responses. On the basis of several experiments, Green, Stevens, and Kuhl (1994) concluded that continuity of fundamental frequency $(f 0)$, but not continuity of formant structure, determined the effective length of the following vowel. In an extension of those efforts, we found here that both effects were critically dependent on particular $f 0$ s and formant values. First, discontinuity in $f 0$ does not necessarily preclude the vowel length effect because the effect maintains when $f 0$ changes from 200 to $100 \mathrm{~Hz}$, and $200-\mathrm{Hz}$ partials extend continuously through test syllables. Second, spectral discontinuity does preclude the vowel length effect when formant changes result in a spectral peak shifting to another harmonic. The results indicate that the effectiveness of stimulus changes for sustaining or diminishing the vowel length effect depends critically on particulars of spectral composition.
\end{abstract}

For any perceptual modality, information about one distal object or event is frequently confounded with information arising from other sources. In vision, for example, the view of one object often is interrupted or concealed by another. Likewise for audition, it is often the case that information for a single acoustic source is obscured by the effect of other acoustic energy produced by competing sound sources. If the resulting percept is to even approximate veridical, then the information from these multifarious sources must be separable. In audition this task seems especially formidable because the air pressure changes caused by multiple sound-producing sources are summated into one sound pressure wave at the ear. In order to give rise to adaptive behavior in the typical environment with competing sound sources, the auditory system must exploit spectral, temporal, and phase information to segregate distal sources.

Presumably, auditory source segregation operates using ecologically principled information about the independence of sources. For instance, if two sources are independent, ${ }^{l}$ one may expect that the resulting sound structures will be independent. That is, sounds that result from different distal sources tend not to start and stop simul-

This research was supported by NIDCD Grant DC-00719 and National Science Foundation Young Investigator Award DBS-9258482 to K.R.K. These data were presented at the fall meeting of the Acoustical Society of America, November 1994, in Austin, Texas. The authors would like to thank Lori Holt for her help in data collection and editing of this manuscript. Correspondence should be addressed to A. J. Lotto, Department of Psychology, 1202 W. Johnson St., Madison, WI 53706 (e-mail: alotto@macc.wisc.edu). taneously; they are usually not harmonically related; they do not often share the same spectral composition; and they typically have different interaural phase relations (i.e., they come from different places in space). Conversely, most correlations between stimulus attributes can be taken as evidence that the attributes emanate from the same source.

The question for researchers is which, if any, of these physical acoustic attributes do human listeners use for source segregation and how does the perceptual system utilize such information in light of other information (e.g., experience or tacit knowledge)? Previous research has demonstrated that humans appear to use many of these attributes for source segregation. Sounds that have asynchronous onsets and offsets tend to be separated perceptually (Bregman \& Pinker, 1978; Vicario, 1973). Harmonically related sounds show greater perceptual grouping than do inharmonic sets (Bregman \& Doehring, 1984; Vicario, 1982). An increase in the frequency separation between two sounds increases their perceptual segregation (Bregman, 1971; Miller \& Heise, 1950; Van Noorden, 1975), as does spatial or lateral separation (Van Noorden, 1975). Finally, co-occurring modulations in amplitude or frequency result in perceptual grouping of the components (Bregman, Abramson, Doehring, \& Darwin, 1985; also see Bregman, 1990, for a comprehensive review of the literature on perceptual grouping in the auditory system).

Despite copious amounts of research about auditory source segregation, there remain many unanswered questions. Many of these earlier studies employed fairly simple stimuli, often using sine-wave tones or combinations of a few tones. In addition to concerns related to the phys- 
ical impoverishment of such sounds (see, e.g., Fowler, 1990), it is also unclear how experience with or knowledge about the stimulus and functionality of the stimulus influences source segregation. Sine-wave tones are not often encountered by subjects outside of the laboratory, and pure tones rarely serve a clear function for human listeners. Consequently, subjects bring little experience or knowledge to bear on the segregation of these sound complexes. Bregman (1990) has suggested that knowledge and experience can be very important for organizing one's auditory world. He has contrasted knowledge-laden "schema-based segregation," with more primitive "primary auditory stream segregation," which is considered automatic and encapsulated. In order to study the contributions of these two proposed classes of segregation processes, one would need to use stimuli with which subjects have experience and which typically require source segregation under normal listening conditions. One type of stimuli that meets these requirements, and that has the benefit of being ecologically relevant for humans, is speech.

The study of speech perception affords an ideal opportunity to describe the processes by which source segregation is accomplished. The perceiver of speech often must contend with interfering extraneous sounds such as door slammings, background music, air conditioner rumblings, and most insidiously, multiple speakers. Communication would be greatly hampered if the listener were to incorporate such incidental sounds into the intended message. The fact that speech is remarkably intelligible even in noisy environments suggests that processes that are responsible for source segregation are very much in evidence during speech perception. As a stimulus set, speech sounds offer many advantages over simpler stimuli such as sine-wave tones. Relative to perception and identification of tones and noise bursts, perception and identification of speech sounds is a very natural and ecologically significant task for subjects. And, not incidentally, the results of experiments using speech have a very immediate and obvious application for automatic speech recognizers and clinical applications for communicative disorders.

Speech sounds have been used successfully to reveal many of the processes involved in source segregation and spectral integration. Many of the same attributes that have been demonstrated to be important for segregation of simple sounds also appear to play a role in segregation and grouping of speech sounds and are of especial utility in segregating the speech of contemporaneous speakers. Again, asynchrony of onsets and inharmonicity have been found to promote segregation of the components (Cutting, 1976; Darwin, 1981; Darwin \& Gardner, 1986; Scheffers, 1983). Spectral distance and trajectories of spectral change greatly influence speech source integration and segregation (Dorman, Cutting, \& Raphael, 1975). Separation in space has also resulted in less integration in several studies employing speech (Cherry \& Taylor, 1954; Huggins, 1964; Schubert \& Schultz, 1962). However, integration of lateralized speech components, which are presumably perceived as arriving from sepa- rate spatial locations, has been demonstrated (Broadbent, 1955; Broadbent \& Ladefoged, 1957; Cutting, 1976; Rand, 1974).

It also bears note that one may not wish to imply that cohesion of phonetic perception relies solely on these sorts of relatively primitive acoustic characteristics. For example, Cutting (1976; Cutting \& Day, 1975) has recommended that in order to account for results from dichotic listening experiments with speech signals, one may wish to incorporate putative processes such as phonetic feature fusion and phonological fusion in addition to sound localization, psychoacoustic fusion, spectral fusion, and spectral/temporal fusion. More recently, Remez and his colleagues (e.g., Remez, Rubin, Berns, Pardo, \& Lang, 1994) have argued that phonetic perceptual organization also requires "specific sensitivity to the acoustic modulations characteristic of speech signals" (p. 129) in order to account for the performance of those subjects who succeed in rendering a phonetic interpretation of sine-wave replicas of spoken sentences.

Aside from the research using sine-wave replicas of speech sounds, many of these previous speech experiments have been concerned primarily with the segregation of simultaneous speech sounds. Experiments described in this paper will exploit a phenomenon referred to here as the "vowel length effect" to determine which attributes are employed by listeners to segregate sequential information about sound sources. In addition, the results will be relevant to the applicability of "schemabased" versus "primary auditory" source segregation processes.

\section{The Vowel Length Effect}

Miller and Liberman (1979) reported a robust effect of vowel duration on the perception of a preceding consonant. They synthesized a series ranging perceptually from $/ \mathrm{ba} /$ to $/ \mathrm{wa} /$ by manipulating the duration of initial formant transitions. When the length of the steady state $\mathrm{la} /$ was changed, the identification boundary between " $b$ " and " $w$ " also changed. Longer vowels resulted in a boundary shift toward longer transition durations (i.e., more "b" responses). In a study of speech production, Miller and Baer (1983) found that, as speaking rate increases, the durations of initial transitions produced were decreased somewhat for $/ \mathrm{ba} /$ and considerably for $/ \mathrm{wa} /$. Taken together, these findings have been adduced as evidence that listeners compensate for changing speaking rates by perceiving initial transitions in a fashion that respects syllable duration and structure. That is, it appears that the listener compensates for increased speaking rate (shorter syllable durations) by perceiving the initial transition as longer. Claims that this particular process is specific to the perception of speech have been challenged by later findings from experiments using nonspeech stimulus analogues. Pisoni, Carrell, and Gans (1983) and Diehl and Walsh (1989) have reported similar results using sine-wave analogues of the speech stimuli from Miller and Liberman (1979). In addition, speech stimuli effects have been replicated in infant listeners (Jusczyk, Pisoni, 
Reed, Fernald, \& Myers, 1983), macaques (Stevens, Kuhl, $\&$ Padden, 1988), and budgerigars (Dent, Powell, Pierce, \& Dooling, 1995).

Independently of issues regarding the degree to which such effects are specific to speech, the results of Miller and Liberman (1979) do demonstrate clearly that the perception of an initial consonant can depend significantly on the length of the following vowel. If vowel duration is to be used, what counts as vowel length becomes important. In addition to rate of speech, other factors, including syllable structure, play a role in vowel duration. For the purposes of the present studies, the concern is with regard to processes through which the auditory system integrates only information that is most likely to constitute the remainder of the syllable. Because of its robustness and generality, the vowel length effect (VLE) may provide an excellent vehicle for investigation of sound source segregation processes. If acoustic energy with some structure is concatenated with the vowel in a consonantvowel $(\mathrm{CV})$ and affects the perceived length of the vowel, as measured by a shift in the identification boundary for the preceding consonant, one can assume that the appended sound structure has been perceptually integrated into the speech syllable. With this operational definition in hand, one can manipulate the acoustic structure of the appended sound to determine the effects of various stimulus attributes on integration as the boundary shift for consonant identification becomes a measure of segregation/ integration across the vowel portion of the CV. Appealing aspects of this paradigm are the naturalness of the task and the relationship between the task and perception of speech in typically noisy environments.

The appropriateness of this type of study for speech motivated several experiments by Green et al. (1994). They were interested in determining the variables that were essential in determining the effective length of a vowel for the VLE. In particular, they investigated whether a sharp discontinuity in fundamental frequency $(f 0)$ or formant structure in the vowel could affect the identification of the syllable-initial consonant. For those studies, two series were synthesized that each varied perceptually from $/ \mathrm{bi} /$ to $/ \mathrm{pi} /$ as spoken by a female. One series contained long-duration syllables $(350 \mathrm{msec})$, while the other contained shorter syllables $(118 \mathrm{msec})$. The voicing distinction between $/ \mathrm{bi} /$ and $/ \mathrm{pi} /$ was synthesized by manipulating the time between the start of the syllable and the onset of periodic energy and energy in the region of the first formant. This duration is commonly referred to as "voice-onset time" (VOT). ${ }^{2}$ It had been shown previously that the identification boundary between " $b$ " and " $p$ " also shifts as a function of vowel length (Green \& Miller, 1985). A longer vowel /i/ will result in a boundary shift toward a longer VOT (i.e., more " $b$ " responses). Green et al. (1994) replicated this finding for the female short and long series and compared the boundary values obtained for these series to boundary values for hybrid stimuli containing abrupt changes in synthesizer parameters.

In one experiment, Green et al. (1994) replaced the final $232 \mathrm{msec}$ of the long-series syllables with a differ- ent synthesized /i/ via digital editing. This appended vowel was synthesized with an $f 0$ contour appropriate for a male speaker, but with the formant values that had been used as synthesis parameters for the long and short female series. Thus, these new hybrid syllables were as long as the long-series ( $350 \mathrm{msec})$ syllables, but contained an abrupt discontinuity in $f 0$ after $118 \mathrm{msec}$, the length of the short-series syllables. The identification boundary for these $350-\mathrm{msec}$ hybrid syllables was not statistically different from that for the short series $(118 \mathrm{msec})$. That is, subjects appeared not to integrate across a change in $f 0$. Segregation and integration of speech based on $f 0$ has also been reported in earlier studies employing different paradigms (Brokx \& Noteboom, 1982; Darwin \& Bethell-Fox, 1977; Scheffers, 1983).

In a second experiment, Green et al. (1994) created another hybrid series by replacing the final $232 \mathrm{msec}$ with a vowel that varied from the rest of the syllable only in formant frequency values. In this hybrid series, syllables had a continuous $f 0$ contour, but changed from formant frequencies modeled after a female speaker's production to those measured for a male's production of the vowel /i/. In this case, the identification boundary was equivalent to that obtained for the long series. Subjects apparently integrated across the formant frequency discontinuity in the hybrid series. Green et al. (1994) concluded that the mechanisms responsible for the VLE are sensitive to $f 0$ changes, but not to spectral discontinuity. This was somewhat surprising because earlier studies had demonstrated the importance of spectral continuity in the segregation/integration of speech (Cole \& Scott, 1973; Dorman et al., 1975; Thomas, Hill, Carroll, \& Garcia, 1970). However, these studies changed phonemic identity as well as spectral continuity.

Perhaps more surprising is that subjects in Green et al. (1994) reported hearing a change in speaker for the hybrid series of both experiments. Either a change in $f 0$ or a change in formant structure was sufficient to signal a change in speaker for these listeners, but only a change in $f 0$ contour was successful in inhibiting integration as indicated by the VLE. This suggests that the phenomenal experience of a change in speaker plays no appreciable role in sound source segregation.

The experiments in the present paper were performed in an attempt to clarify and extend the results of Green et al. (1994). In Experiment 1, we examined the effects of harmonicity and $f 0$ change on the integration of speech signals. In Experiment 2, we distinguished the effects of phonemic identity from effects of spectral change for sound source segregation. We hope that, together, these experiments will elucidate some of the attributes utilized by the auditory system for source segregation and demonstrate the utility of this paradigm for future research.

\section{EXPERIMENT 1}

The first experiment was an attempt to clarify the effect of $f 0$ discontinuity on the VLE. There are several potential explanations for the results obtained in the $f 0$ 
experiment of Green et al. (1994). Stimuli used by Green et al. confounded $f 0$ discontinuity and $f 0$-contour discontinuity. For their hybrid stimuli, $f 0$ increased from 180 to $206 \mathrm{~Hz}$ over the first $50 \mathrm{msec}$. Then $f 0$ decreased linearly to approximately $196 \mathrm{~Hz}$ at the point at which the second vowel was appended (118 msec from the beginning of the syllable). Over the last $232 \mathrm{msec}$ of the syllable, $f 0$ decreased from approximately 114 to $90 \mathrm{~Hz}$. Thus, at the point of $f 0$ discontinuity, there was a discontinuity in $f 0$ slope from .15 to $.10 \mathrm{~Hz} / \mathrm{msec}^{3}{ }^{3}$ To distinguish possible effects of $f 0$ frequency and of contour discontinuity, the stimuli in Experiment 1 were synthesized with a flat $f 0$ contour.

Another possible explanation for the results of Green et al. (1994) could be based on the spectral effects of changing $f 0$. When $f 0$ is changed, the frequency spacing of the harmonics also changes. Therefore, an $f 0$ discontinuity results in a spectral discontinuity. While the nominal frequency values of the formants (synthesizer parameters) remain the same, physical peaks in the spectrum are shifted. As a consequence, an impediment to integration could be spectral discontinuity and not discontinuity in perceived pitch as specified by $f 0$. To test this possibility, a hybrid series was created in which the change in $f 0$ was a perfect octave, from 200 to $100 \mathrm{~Hz}$. In this way, the actual harmonics were not shifted; more were simply added. If subjects show evidence of integration across this change, then a simple explanation based only on pitch tracking may prove insufficient.

\section{Method}

Subjects. Fifteen undergraduates at the University of WisconsinMadison participated in the experiment for course credit. None of the subjects reported hearing disorders and all were native speakers of English.

Stimuli. Stimuli were based on those used in Green et al. (1994) with some modifications to create a flat $f 0$ contour. Two 10 -step $/ \mathrm{bi} /-/ \mathrm{pi} /$ series were created using the cascade synthesizer described in Klatt (1980) and implemented on a 486 microcomputer (CSYNTR16; Jamieson, Ramji, Kheirallah, \& Nearey, 1992). Syllables in each series varied in VOT from 15 to $60 \mathrm{msec}$ in $5-\mathrm{msec}$ steps. One series, the long series, consisted of syllables that were $350 \mathrm{msec}$ in duration. The short series consisted of $120-\mathrm{msec}$ syllables. In all aspects other than vowel duration, these two series were identical. Each syllable began with a 5 -msec frication-noise burst followed by formant transitions ( $30 \mathrm{msec}$ for $F 1 ; 45 \mathrm{msec}$ for $F 2$ and $F 3$ ). During the transitions, synthesizer values for $F 1$ increased from $290 \mathrm{~Hz}$ to the steady-state value of $376 \mathrm{~Hz}$; for $F 2$ they increased from 1629 to $2508 \mathrm{~Hz}$; for $F 3$ they increased from 2450 to $3100 \mathrm{~Hz}$; and for $F 4$ they remained at $3300 \mathrm{~Hz}$ throughout the stimulus. During the aspirated portion of the syllable (from initial noise burst to onset of periodic energy), formant filters were excited by a noise source (synthesizer parameter $\mathrm{AH}$ ) and bandwidths of the formants were manipulated. Bandwidth of $F 1$ decreased from 450 to $50 \mathrm{~Hz}$; bandwidth of $F 2$ decreased from 170 to $100 \mathrm{~Hz}$; bandwidth of $F 3$ decreased from 200 to $130 \mathrm{~Hz}$; and bandwidth of $F 4$ remained at $250 \mathrm{~Hz}$ throughout the stimulus. All syllables had a constant $f 0$ of $200 \mathrm{~Hz}$.

In addition to the short and long series, two hybrid series were created by replacing the last $230 \mathrm{msec}$ from the syllables of the long series with either of two different steady-state vowels using a digital waveform editing program (CSRE; Jamieson et al., 1992). Synthesis parameters for the two appended vowels were identical ex- cept for $f 0$. Formant-frequency and bandwidth synthesizer values were the same as those used for the steady-state portion of the long and short series. The appended vowel of the harmonic series had an $f 0$ of $100 \mathrm{~Hz}$. Thus, the two $f 0 \mathrm{~s}$ of the harmonic stimuli were a perfect octave apart. For the inharmonic series the $f 0$ changed from 200 to $114 \mathrm{~Hz}$ after $120 \mathrm{msec}$. This is approximately the $f 0$ difference at the point of discontinuity in the Green et al. (1994) stimuli.

Care was taken to assure that no amplitude discontinuities occurred due to the waveform editing. The appended vowel was matched in RMS level to the steady state of the long-series syllables. Digital splicing was performed at zero crossings of the waveform and periods of the fundamental were left intact.

The stimuli are presented in schematized form in Figure 1. As can be seen from these pseudo-spectrograms, the syllables of the hybrid series were of the same duration as the syllables of the long

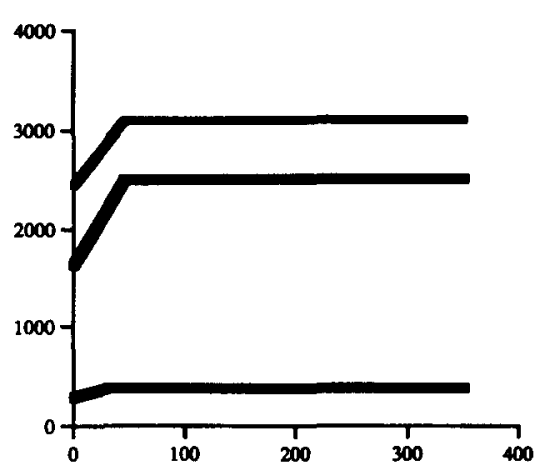

Long

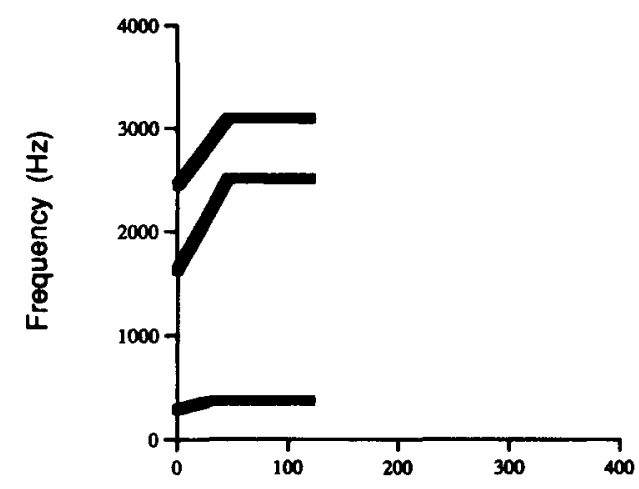

Short

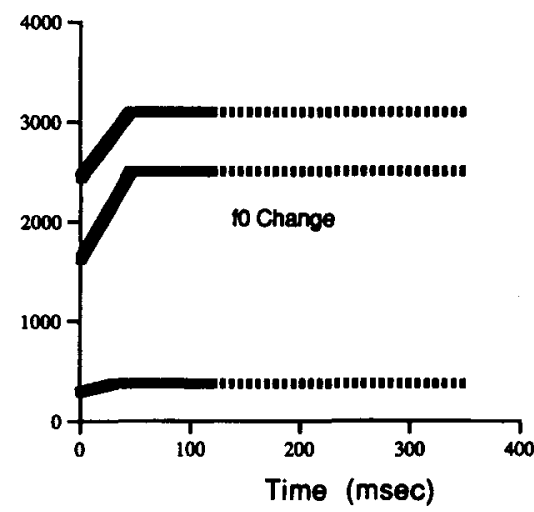

Hybrid

Figure 1. Synthesizer formant frequency values $(F 1, F 2, F 3)$ for Experiment 1 stimuli plotted across time. Note that the $f 0$ change in the hybrid stimuli occurs 120 msec into the syllable, which is the duration of the short stimuli. 
series, but the hybrid-series syllables had $f 0$ discontinuities after $120 \mathrm{msec}$, which was the duration of the short-series syllables.

Stimulus presentation and response collection were under the control of a 486-25 microcomputer. Following D/A conversion (Ariel DSP-16), stimuli were low-pass filtered $(4.8-\mathrm{kHz}$ cutoff frequency, Frequency Devices 677), amplified (Stewart HDA4), and presented to subjects via Beyer DT-100 headphones.

Procedure. One to 3 subjects were run at one time in three singlesubject soundproof chambers (Suttle Equipment Corp.) during a single experimental session consisting of two 15 -min test periods. During the first test period, subjects heard all members of the long and short series 10 times each ( 200 trials). The second test period consisted of the members of the harmonic and the inharmonic series presented 10 times each. Subjects always heard the long and short series first because it was thought that experience with these basic stimuli would facilitate the identification of the less natural hybrid stimuli. Within a test period, stimuli were presented in random order at $75 \mathrm{~dB}$ SPL(C) at a rate of about one stimulus every $3 \mathrm{sec}$. Subjects were instructed to identify the syllable-initial consonant by pressing either of two buttons labeled "bee" and "pee."

\section{Results}

One subject's data were withheld from further analysis because the subject used only one of the possible labels in identifying the hybrid-series stimuli. The remaining 14 individual identification boundaries were determined for each series using probit analysis. ${ }^{4}$ The mean boundary values for each series are displayed in Figure 2. An analysis of variance (ANOVA) performed on these values revealed a significant effect of series type $[F(3,39)=$ $9.12, p<.0005]$. Post hoc analyses (Tukey least significant difference tests with $\alpha=.05$ across all comparisons) demonstrated that the vowel length effect was replicated in that the boundary associated with the long series $(41.06 \mathrm{msec})$ was significantly longer than the boundary for the short series $(29.57 \mathrm{msec})$. As reported in earlier studies, an increase in vowel length resulted in

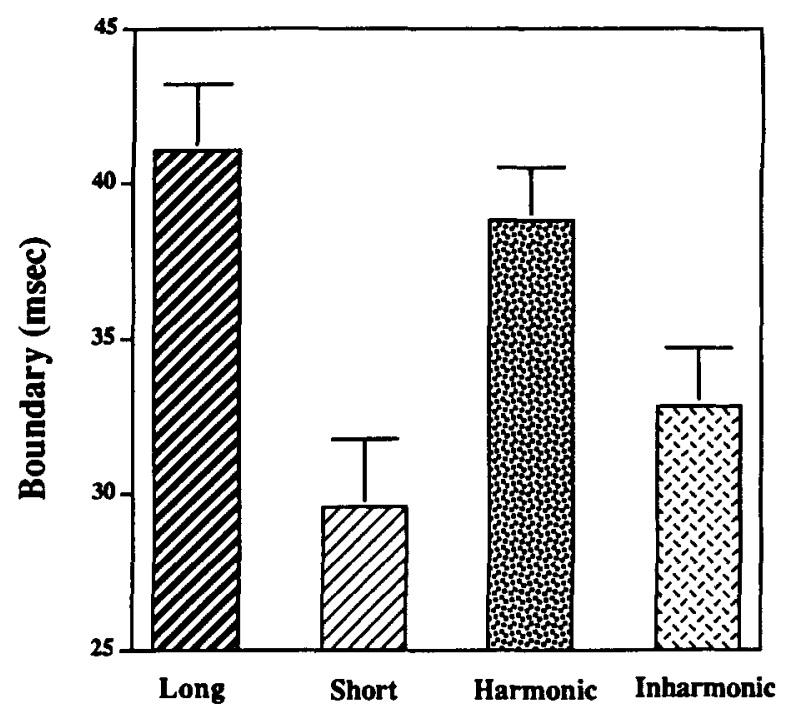

\section{Stimulus Series}

Figure 2. Mean boundary values with attendant standard errors for each stimulus series in Experiment 1. a boundary shift toward longer VOTs. The identification boundary $(32.82 \mathrm{msec})$ for the series with a midvowel shift from 200 to $114 \mathrm{~Hz}$, the inharmonic series, differed significantly from the long-series boundary, but did not differ significantly from the short-series boundary, suggesting that subjects failed to integrate over the $f 0$ discontinuity even in the absence of an $f 0$-slope discontinuity, since subjects responded as if the inharmonic syllables had been $120 \mathrm{msec}$ long. Taken alone, this result is ostensibly consistent with the conclusion of Green et al (1994) that the mechanism responsible for the VLE is sensitive to changes in $f 0$. In contrast, the harmonic series, which included a larger $f 0$ discontinuity in terms of absolute frequency difference $(200-100 \mathrm{~Hz})$, had a significantly longer boundary value $(38.81 \mathrm{msec})$ than did that associated with the inharmonic series. The boundary for the harmonic series was also significantly different from the boundary for the short series, but did not differ significantly from the long-series boundary. Thus, subjects integrated across the harmonic shift in $f 0$ and responded as if harmonic syllables had been $350 \mathrm{msec}$ long.

The dichotomy between the results for the two hybrid series suggests that processes responsible for the VLE do not rely solely on $f 0$ for segregation of speech sound sources across time. Subjects perceptually integrated across the $100-\mathrm{Hz} f 0$ discontinuity in the harmonic stimuli, which was the larger of the two $f 0$ discontinuities presented. Presumably this disparity between the two hybrid series arises from the octave relation between the two $f 0$ s of the harmonic stimuli. It is possible that the perceptual system regards harmonic relations as evidence of an equivalent genesis for separate sounds. Components arising from a single periodic vibrating source tend to be harmonically related. This could certainly be an ecologically valid cue for attributing several sound components to a single source. Previous research has established that harmonicity increases the integration of sounds (Bregman \& Doehring, 1984; Darwin, 1981; Vicario, 1982). However, these efforts were concerned primarily with integrating simultaneous harmonic components. The present study concerns integration of serial, adjacent sounds. The proposed ecological explanation related above is not as obvious in this case. It is not readily apparent why sounds presented from a single source across time should have harmonically related $f 0$ s. It would seem that a more reliable signal for the presence of a single source would be continuity of the $f 0$ contour. Nevertheless, in the present study segregation by $f 0$ was clearly ameliorated by the octave relation.

A second plausible explanation for the current pattern of results is that perceptual processes of source segregation utilize general spectral discontinuities. All of the harmonics of the first $120 \mathrm{msec}$ of the harmonic stimuli (multiples of $200 \mathrm{~Hz}$ ) were present in the last $230 \mathrm{msec}$ (multiples of $100 \mathrm{~Hz}$ ), while in the inharmonic stimuli there were no common harmonics below the filter cutoff frequency of $4.8 \mathrm{kHz}$ between the two parts of the stimuli. This shift in frequency components also results in a shift in the overall pattern of amplitude values across the 
harmonics. Such a global spectral discontinuity could be a cue that there are two disparate sound sources. The use of spectral discontinuities for source segregation is examined in Experiment 2.

\section{EXPERIMENT 2}

Green et al. (1994) reported that spectral discontinuities do not play an appreciable role in determining the effective length of a vowel for the VLE. They created a series that contained an abrupt shift in nominal synthesizer formant frequency values. The identification curves that they obtained indicated that subjects perceptually integrated across the formant value changes. In light of these results, Green et al. concluded that continuity of formant structure was not an important determinant of vowel length. However, an alternative conclusion is that the formant changes used by Green et al. were not drastic enough to effect a change in perception. The largest discontinuity in nominal formant values tested by Green et al. included a shift in the synthesizer frequency parameter for $F 1$ from 313 to $376 \mathrm{~Hz}$. The $f 0$ for these stimuli at the point of discontinuity was approximately $196 \mathrm{~Hz}$. Thus, the frequency of the peak harmonic (i.e., the harmonic with the largest amplitude) in the region of $F 1$ did not change at the point of discontinuity. The second harmonic $(392 \mathrm{~Hz})$ remained the peak harmonic for synthesizer values of 313 and $376 \mathrm{~Hz}$. The Green et al. hybrid syllables did contain a shift in the frequencies of the peak harmonics in the region of $F 2$ and $F 3$, but it is questionable whether these constituted a salient change because formant peaks for $F 2$ and $F 3$ are close and can be relatively undifferentiated for an $/ \mathrm{i} / .^{5}$ Perhaps the shifts in nominal formant frequency values did not engender a large enough spectral discontinuity to hamper the integration of these sequential sounds.

Experiment 2 was designed to test the effects of a larger spectral discontinuity on the VLE. For this experiment, a hybrid series for which syllables contained a sudden discontinuity of nominal formant values was created (peakshift). This shift in formant values was large enough to engender a shift in the peak harmonics $120 \mathrm{msec}$ into the syllable. As a control, a second hybrid series (peakconstant) was created that included a discontinuity in nominal formant parameters, but no shift in the identity of the peak harmonic, as was the case for the stimuli used in Green et al. (1994). In contrast to the syllables of peakshift and the stimuli used in Green et al., the peak-constant syllables were designed to elicit a change in phonemic identity at the point of formant structure discontinuity. Consonant identification boundaries for these hybrid series and the results of Green et al., together, should shed light on the influence of phonemic identity and formant frequency structure on integration and segregation of speech sounds.

\section{Method}

Subjects. Twenty-six undergraduates at the University of Wisconsin-Madison participated in the experiment for course credit. None of the subjects reported hearing disorders and all were native speakers of English.

Stimuli. In order to effect a shift in the peak harmonic with a small enough change in the frequency of $F 1$ to maintain phonetic identity, the stimuli were synthesized with a relatively low $f 0$ of $100 \mathrm{~Hz}$. A long series with 350 -msec syllables and a short series with 120 -msec syllables were again synthesized. The syllables were similar to those used in Experiment 1 except for a change in the nominal formant frequency values to better reflect a male vocal tract. During the transitions of these syllables, synthesizer parameters for $F 1$ increased from 200 to $328 \mathrm{~Hz}$; for $F 2$ they increased from 1300 to $2135 \mathrm{~Hz}$; for $F 3$ they increased from 2150 to $2700 \mathrm{~Hz}$; and for $F 4$ they remained at $3300 \mathrm{~Hz}$ throughout the stimulus.

Again, two hybrid series were created by replacing the final $230 \mathrm{msec}$ of the long-series syllables with a slightly altered steadystate vowel. For the peak-constant series, the appended vowel had a nominal $F 1$ of $349 \mathrm{~Hz}$, an $F 2$ of $2085 \mathrm{~Hz}$, an $F 3$ and an $F 4$ of $2700 \mathrm{~Hz}$ and $3300 \mathrm{~Hz}$, respectively. The value for $F 1$ for the peakshift series was $200 \mathrm{~Hz}$; for $F 2$ it was $2500 \mathrm{~Hz}$; for $F 3$ it was $3010 \mathrm{~Hz}$; and for $F 4$ it was $3400 \mathrm{~Hz}$. Note that the frequency of the peak harmonics did not change across the discontinuity for the peakconstant stimuli, but that the change in formant frequency across the discontinuity of the peak-shift stimuli did result in a switch of the peak harmonic of each of the four formants. In particular, the peak harmonic in the area of $F l$ changed from the third harmonic
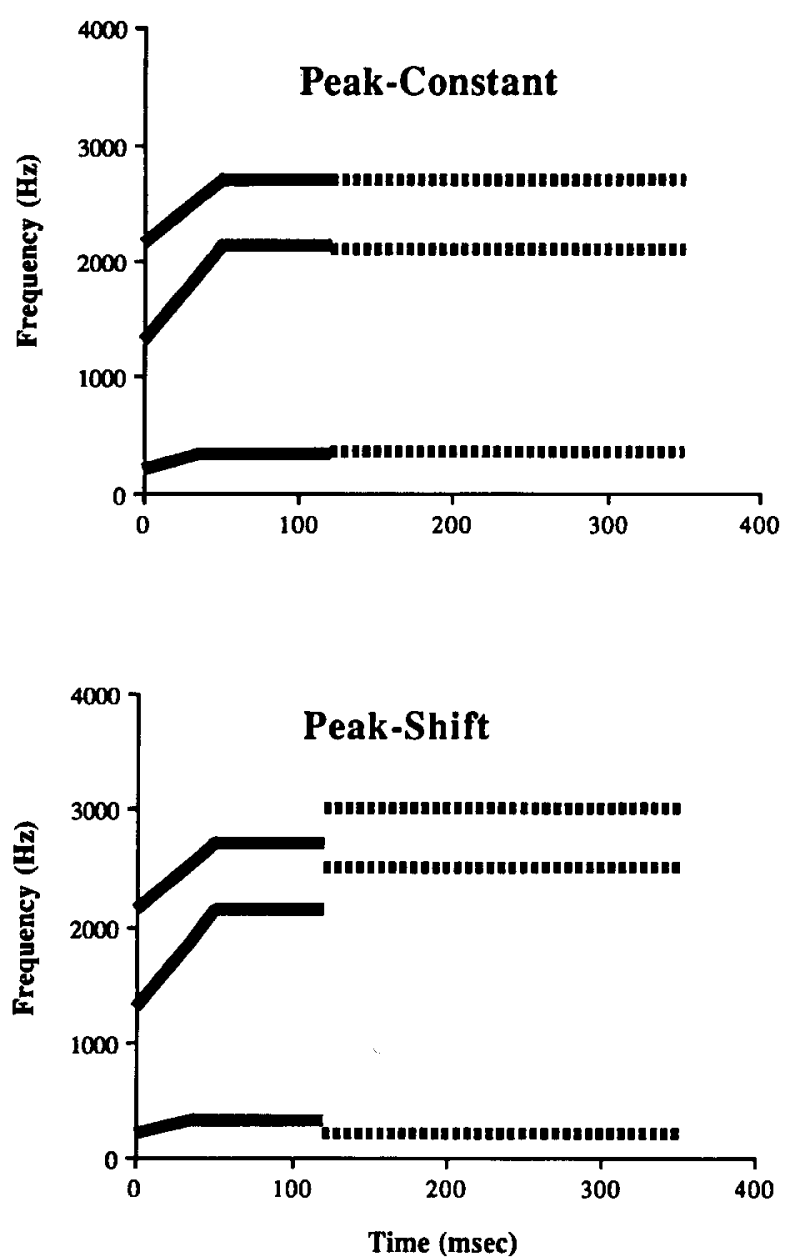

Figure 3. Synthesizer formant frequency values $(F 1, F 2, F 3)$ for the hybrid series of Experiment 2 plotted across time. 
$(300 \mathrm{~Hz})$ to the second harmonic $(200 \mathrm{~Hz})$. Schematic spectrograms of the hybrid series are displayed in Figure 3.

Of course, such a large change in nominal formant frequency values may lead to a shift in the perceived phonemic identity of the segment. Formant values of the appended vowels in the hybrid series were selected carefully to account for this possibility. The steady state of the short and long series was determined to be a reasonable exemplar of $/ \mathrm{i} /$. The more extreme shift in formant frequency values (peak-shift) created a vowel with a higher $F 2$ and a lower $F 1$ (more/i/like). The smaller shift in formant frequency (peak-constant) was in the direction of the $/ \mathrm{i} /-/ \mathrm{I} /$ boundary (i.e., a lower $F 2$ and a higher $F 1$ ). Thus, the smaller change in formant values was expected to be more likely to result in a change in phonemic identity. To assure that this manipulation was successful, subjects were also asked to identify the vowels included in this experiment. A separate set of stimuli was created for the vowel identification task. Instead of presenting each steady-state vowel in isolation, the vowels were presented in context. One of the stimuli consisted of $120 \mathrm{msec}$ of the steady-state vowel from the short and long series (the base vowel) digitally spliced to precede $230 \mathrm{msec}$ of the appended vowel of the peak-constant stimuli (base/constant). Another consisted of the base spliced to $230 \mathrm{msec}$ of the appended vowel of the peakshift series (base/shift). The final two stimuli for vowel identification were inverses of the two previously described. One stimulus was constructed by splicing the $120 \mathrm{msec}$ of the steady state from the long series at the end of the appended vowel from the peak-constant stimuli (constant/base). The other was constructed in a similar manner using the appended vowel from the peak-shift series (shift/ base). Again, care was taken to assure that the phase of $f 0$ was maintained and that the vowels were matched in RMS amplitude.

Procedure. The procedure for the identification of the initial consonant of the four series was identical to that in Experiment 1. In addition, prior to the consonant identification task, subjects participated in a vowel identification task. Randomized blocks of the four vowel pairings were played 10 times each. Subjects were asked to identify the last vowel of the pair (or the only vowel if they perceived just one vowel) as an exemplar of the vowel in "beat," "bit," "bet" or "book." The vowels / $/$ and /U/ were used as alternative labels because they are close neighbors in the English vowel space. For this task, subjects used a labeled four-button response box.

\section{Results}

Seven subjects' data were withheld from further analysis because they failed to use one of the possible labels for identifying the initial consonants of the hybrid-series stimuli. Presumably the unnaturalness of the hybrid stimuli gave some subjects difficulty in the labeling task.

Vowel identification. The vowel identification task was performed to test whether or not the subjects perceived a phonemic identity discontinuity in the hybrid series. In order to answer this question, the percentage of blocks with identical categorizations of the base and appended vowels was calculated. For the peak-constant stimuli, this is the percentage of blocks in which base/constant and constant/base were identified with the same category label. For the peak-shift stimuli, this is the percentage of blocks in which base/shift and shift/base were categorized the same. Percentages with error bars are presented in Figure 4.

As expected from the design of the stimuli, vowels constituting the peak-shift stimuli were more often identified as phonemically identical $(67.37 \%)$ than were the vowels constituting the peak-constant series $(52.63 \%)$.

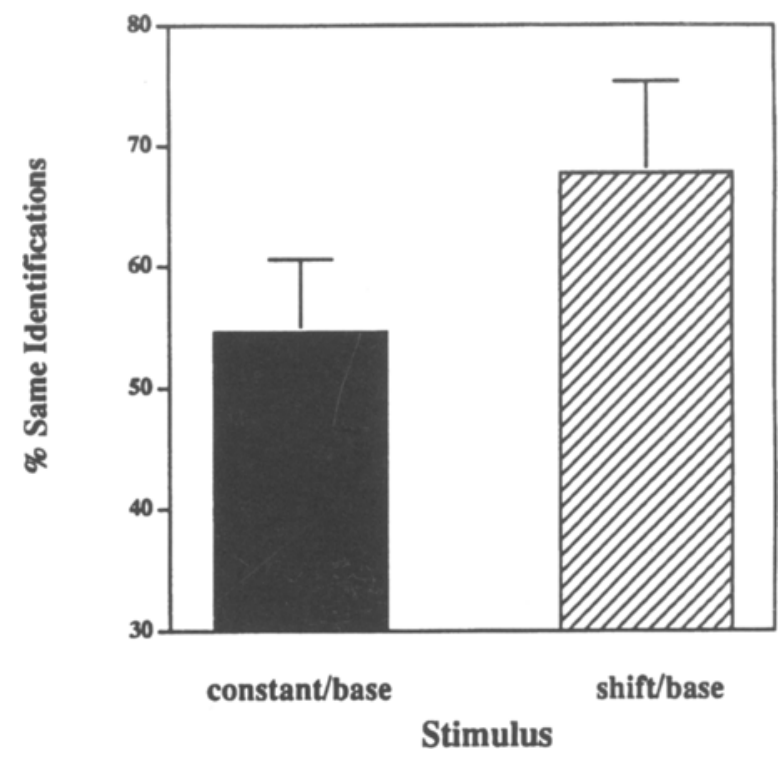

Figure 4. Percentage of blocks containing equivalent identifications for the two vowel steady states in each of the hybrid series.

Consonant identification. Individual consonant identification boundaries were determined for each series using probit analysis. Mean boundary values are displayed in Figure 5. An ANOVA performed on the boundaries revealed a significant effect of series type $[F(3,54)=11.52$, $p<.0001]$. The pattern of results was similar to that obtained in Experiment 1. Once again, identification boundaries for the long series $(39.28 \mathrm{msec})$ were significantly longer (Tukey tests with $\alpha=.05$ across all of the comparisons) than those obtained for the short series $(34.63 \mathrm{msec})$. This is another demonstration of the VLE. This effect size was smaller than that obtained for Experiment 1. This is most probably due simply to the details of stimulus construction with the series in Experiment 2 being modeled after different productions from those in Experiment 1.

Replicating the results of Green et al. (1994), boundaries associated with the peak-constant $(/ \mathrm{i} /-/ \mathrm{I} /)$ series ( $38.99 \mathrm{msec})$ were significantly longer than the boundaries for the short series, but were not statistically different from those for the long-series boundaries. When presented with a small spectral discontinuity, subjects perceptually integrated the appended vowel to the rest of the syllable. In contrast, when a large spectral discontinuity occurred in the stimulus, integration was effectively blocked. The boundaries for the peak-shift $(/ \mathrm{i} /-/ \mathrm{i} /)$ series $(33.27 \mathrm{msec})$ were significantly shorter than were the boundaries for the long series, but were indistinguishable from the boundaries of the short series. It is interesting that syllables for which subjects were more likely to perceive a midsyllable shift in vowel identity (peak-constant) were also more likely to be treated as cohesive wholes with regard to determining the identity of the initial consonant. 


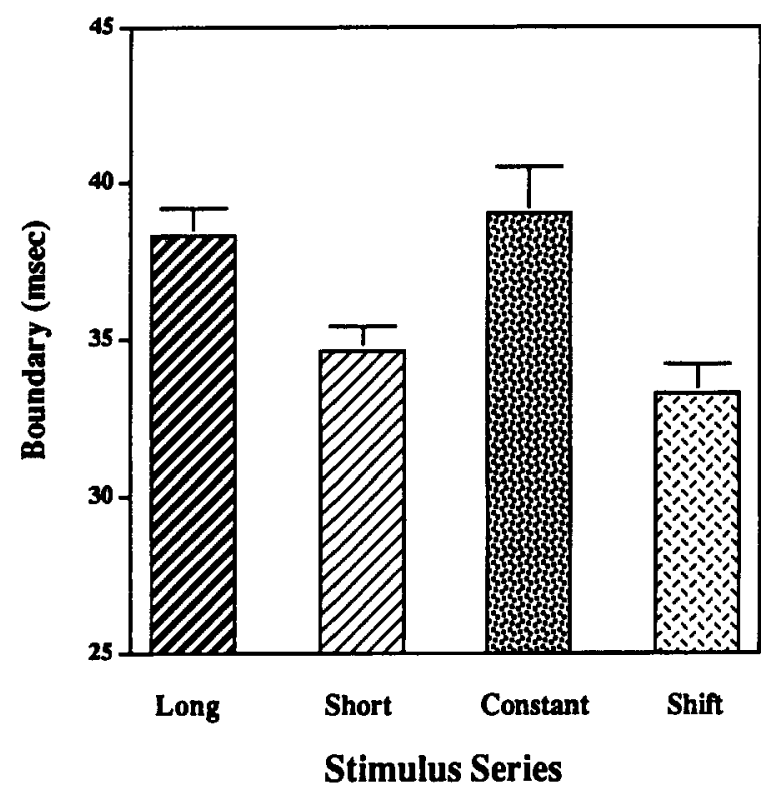

Figure 5. Mean boundary values with attendant standard errors for consonant identification of each stimulus series in Experiment 2.

It may be possible that subjects' identification of the vowels could have affected their identification of the consonants, and that such a correlation was obscured in the summarizing of data across subjects. The correlations between the percentage of equivalent identifications for the vowels of the hybrid series and the boundary value of the consonant identifications for each subject were also calculated and subjected to a $t$ test. For neither series was the correlation significant and, in fact, it was slightly negative for both [peak-constant: $r=-.24, t(18)=-1.04$, $p=.31$; peak-shift: $r=-.08, t(18)=-0.31, p=.76]$. Perceived phonemic continuity was not predictive of the identification of the initial consonant.

In light of all these results, the perceptual ineffectiveness of the formant manipulation in Green et al. (1994) on the VLE appears to be due to the small extent of the resulting spectral contrast. When a formant-value discontinuity results in a shift of the peak harmonics, integration is hampered.

\section{GENERAL DISCUSSION}

The present two experiments serve to clarify and extend the results of Green et al. (1994). On the basis of their findings, Green et al. (1994) concluded that $f 0$ played a critical role in the determination of syllable length for the VLE, but formant structure did not. The results of Experiment 1 in the present report demonstrate that a large discontinuity in $f 0$, per se, is not sufficient to shorten the effective length of the syllable. When the $f 0$ change was an octave (e.g., 200-100 Hz), subjects perceptually integrated over the $f 0$ discontinuity. On the other hand, a smaller inharmonic discontinuity in $f 0(200-114 \mathrm{~Hz})$ disrupted the integration of sequential information.
Likewise, the results of Experiment 2 demonstrated that the effects of formant structure on the VLE depend more on spectral composition than was suggested by Green et al (1994). Syllables that contained larger spectral discontinuities were identified as if the vowel length ended at the discontinuity. The divergence in results from the present paper and Green et al. is probably due to the difference in the extent of the spectral discontinuity used. The nominal formant changes used for the synthesis of the stimuli in Green et al. did not result in a shift of the frequency of the peak harmonic around $F 1$. In Experiment 2 of the present report, the two sets of hybrid stimuli differed in degree of spectral discontinuity, and the series that included shifts in the peak harmonics was identified in a similar manner to the short series; that is, segregation of serial sounds occurred.

Of additional interest was the lack of effect of perceived phonemic identity on the results in Experiment 2. In addition to the absence of speaker effects demonstrated in Green et al. (1994), this shows a surprising dearth of causal efficacy for "higher order" perceptual qualities. Both of these results are suggestive of processes that are relatively stimulus driven and automatic. However, neither Green et al. (1994) nor the present studies included a measure of speaker change or phonemic change that was concurrent with the consonant-labeling task. It remains to be shown what the phenomenal experience is during the VLE task.

It does not seem unreasonable to suggest that the sound integration/segregation process that determines the effective length of a syllable for the VLE is a general sound source segregation process that has come to serve adaptively in the ecology of sound-producing objects and events. Here, it is presumed that a perceptual system must be adapted to segregate information by source to some extent. In general, an organism should be able to parcel information from external objects and events to the degree that this separate information encourages different adaptive actions. Constraints of the external world are for the most part regular, and plausible configurations of sound sources are limited by laws governing mass and inertia. Kluender (1991), for example, has suggested that the physical attribute, limited elasticity, serves to constrain the changes a sound-producing source can undergo over some period in time. This suggestion is similar in character to Ullman's (1984) suggested rigidity constraint for recovery of 3-D shape by the visual system. For the case of acoustics, most physical objects cannot change their shape greatly instantaneously. ${ }^{6}$ Changes in shape occur across time with an integration of intermediate shapes. What does this mean for the resulting sounds that physical objects produce? In the cases of a source like the vocal chords or a resonator like the vocal tract, this means that sounds arising from one source will not make large, instantaneous shifts in spectral-temporal character. Sounds produced by a single source will tend to smoothly change or remain steady state. Contiguous intervals of these sounds will tend to be correlated. In contrast, independent sources 
are not identically constrained by the physical laws of inertia and mass and their respective sounds will tend not to be correlated.

The present experiments demonstrate that sounds with large instantaneous changes in spectral composition tend to give rise to perceptual segregation. When the harmonics of peak amplitude were changed, listeners did not integrate across the discontinuity. This instantaneous peak harmonic shift was accomplished by formant frequency value changes in Experiment 2 and by an inharmonic change in $f 0$ in Experiment 1 . These kinds of instantaneous shifts in spectral shape arising from a single sound source would rarely, if ever, occur in the acoustic ecology. This regularity is reflected in the auditory perceptual solutions for the chimerical stimuli presented in these studies. This suggested framework is consistent with "psychoacoustic complementarity" (Kluender, 1991)-a development for the auditory system of Shepard's (1984) "psychophysical complementarity." According to these theories, operating constraints of perceptual systems have been shaped by evolution and experience to reflect the constraints operating on the external world.

The above description is not sufficiently specific to be wholly explanatory. A detailed metric of spectral discontinuity must be developed in order to make more quantitative predictions from this kind of framework. Nevertheless, the framework unifies the results presented here and in Green et al. (1994). One would not expect languagespecific attributes of speech, such as phonemic identity, to have an effect on a general sound source segregation process. The communication system of speech was built on this auditory foundation. By this account, one reason duration-based contrasts in speech could develop is because there was already a mechanism in place that sorted sound by source. Without this segregation, signals based on relative duration would be meaningless in a cluttered acoustic environment.

Further efforts to better determine the attributes important for sound source segregation and how these attributes are related to physical constraints on sound sources would be most welcome. Methodologies like the one used in the present report may serve to reveal mechanisms and stimulus attributes that allow perceptual systems to maintain a coherent view of the external world and to support adaptive actions.

\section{REFERENCES}

BREgman, A. S. (1971). Primary auditory stream segregation and the perception of tunes. Unpublished manuscript, McGill University, Department of Psychology.

Bregman, A. S. (1990). Auditory scene analysis. Cambridge, MA: MIT Press.

Bregman, A. S., Abramson, J., Doehring, P., \& Darwin, C. J. (1985). Spectral integration based on common amplitude modulation. Perception \& Psychophysics, 37, 483-493.

Bregman, A. S., \& Doehring, P. (1984). Fusion of simultaneous tonal glides: The role of parallelness and simple frequency relations. Perception \& Psychophysics, 36, 251-256.

Bregman, A. S., \& Pinker, S. (1978). Auditory streaming and the building of timbre. Canadian Journal of Psychology, 32, 19-31.
Broadbent, D. E. (1955). A note on binaural fusion. Quarterly Journal of Experimental Psychology, 7, 46-47.

BROADBENT, D. E., \& LADEFoged, P. (1957). On the fusion of sounds reaching different sense organs. Journal of the Acoustical Society of America, 29, 708-710.

BroKx, J. P. L., \& NoteBoom, S. G. (1982). Intonation and the perceptual separation of simultaneous voices. Journal of Phonetics, 10, 23-36.

Cherry, E. C., \& TAYLOR, W. K. (1954). Some further experiments upon the recognition of speech, with one and with two ears. Journal of the Acoustical Society of America, 26, 554-559.

ColE, R. A., \& SCOTT, B. (1973). Perception of temporal order in speech: The role of vowel transitions. Canadian Journal of Psychology, 27, 441-449.

CutTING, J. E. (1976). Auditory and linguistic processes in speech perception: Inferences from six fusions in dichotic listening. Psychological Review, 83, 114-140.

Cutting, J. E., \& DaY, R. S. (1975). The perception of stop-liquid clusters in phonological fusion. Journal of Phonetics, 2, 99-113.

DARWIN, C. J. (1981). Perceptual grouping of speech components differing in fundamental frequency and onset-time. Quarterly Journal of Experimental Psychology, 33A, 185-207.

Darwin, C. J., \& BeThell-Fox, C. E. (1977). Pitch continuity and speech source attribution. Journal of Experimental Psychology: Human Perception \& Performance, 3, 665-672.

DARwin, C. J., \& GaRDNER, R. B. (1986). Mistuning a harmonic of a vowel: Grouping and phase effects on vowel quality. Journal of the Acoustical Society of America, 76, 838-845.

Dent, M. L., Powell, E. F., Pierce, A., \& Dooling, R. J. (1995). Discrimination of synthetic /ba-wa/ by budgerigars (Melopsittacus undulatus). Journal of the Acoustical Society of America, 97, $3417(5 p S C 4)$

DIEHL, R. L., \& WALSH, M. A. (1989). An auditory basis for the stimuluslength effect in the perception of stops and glides. Journal of the Acoustical Society of America, 85, 21 54-2164.

Dorman, M. F., Cutting, J. E., \& Raphael, L. J. (1975). Perception of temporal order in vowel sequences with and without formant transitions. Journal of Experimental Psychology: Human Perception \& Performance, 1, 121-129.

FowlER, C.A. (1990). Sound-producing sources as objects of perception: Rate normalization and nonspeech perception. Journal of the Acoustical Society of America, 88, 1236-1249.

GreEN, K. P., \& MiLLER, J. L. (1985). On the role of visual rate information in phonetic perception. Perception \& Psychophysics. 38, 269-276.

Green, K. P., Stevens, E. B., \& Kuhl, P. K. (1994). Talker continuity and the use of rate information during phonetic perception. Perception \& Psychophysics, 55, 249-260.

HUGGINS, A.W. F. (1964). Distortion of temporal patterns of speech: Interruption and alternation. Journal of the Acoustical Society of America, 36, 1055-1065.

Jamieson, D. J., Ramji, K. V., Kheirallah, I., \& Nearey, T. M. (1992). CSRE: A speech research environment. In J. Ohala, T. Nearey, B. Derwing, M. Hodge, \& G. Wiebe (Eds.), Proceedings ICSLP 92 (pp. 1127-1130). Edmonton, AB: University of Alberta Press.

Jusczyk, P. W., Pisoni, D. B., Reed, M. A., Fernald, A., \& Myers, M. (1983). Infants' discrimination of the duration of a rapid spectrum change in nonspeech signals. Science, 222, 175-177.

KLATT, D. K. (1980). Software for a cascade/parallel formant synthesizer. Journal of the Acoustical Society of America, 67, 971-995.

KLuENDER, K. R. (1991). Psychoacoustic complementarity and the dynamics of speech production and perception. Perilus, 14, 131-135.

Miller, G. A., \& Heise, G. A. (1950). The trill threshold. Journal of the Acoustical Society of America, 22, 637-638

Miller, J. L., \& BAer, T. (1983). Some effects of speaking rate on the production of $/ \mathrm{b} /$ and $/ \mathrm{w} /$. Journal of the Acoustical Society of America, 73, 2124-2133.

Miller, J. L., \& Liberman, A. M. (1979). Some effects of later-occurring information on the perception of stop consonant and semivowel. Perception \& Psychophysics, 25, 457-465.

Pisoni, D. B., Carrell, T. D., \& Gans, S. J. (1983). Perception of the duration of rapid spectrum changes in speech and nonspeech signals. Perception \& Psychophysics, 34, 314-322. 
RAND, T. C. (1974). Dichotic release from masking for speech. Journal of the Acoustical Society of America, 55, 678-680.

Remez, R. E., Rubin, P. E., Berns, S. M., Pardo, J. S., \& Lang, J. M. (1994). On the perceptual organization of speech. Psychological Review, 101, 129-156.

SCHEFFERS, M. T. M. (1983). Sifting vowels: Auditory pitch analysis and sound segregation. Unpublished doctoral dissertation, Groningen University.

Schubert, E. D., \& Schultz, M. C. (1962). Some aspects of binaural signal selection. Journal of the Acoustical Society of America, 34, 844-849.

SHEPARD, R. N. (1984). Ecological constraints on internal representation: Resonant kinematics of perceiving, imagining, thinking, and dreaming. Psychological Review, 91, 417-447.

Stevens, E. B., Kuhl, P. K., \& PADDEN, D. M. (1988). Macaques show context effects in speech perception. Journal of the Acoustical Society of America, 84 (Suppl. 1), S77.

Thomas, I. B., Hill, P. B., Carroll, F. S., \& Garcia, B. (1970). Temporal order in the perception of vowels. Journal of the Acoustical Society of America, 48, 1010-1013.

Ullman, S. (1984). Maximizing rigidity: The incremental recovery of 3-D structure from rigid and nonrigid motion. Perception, 13, 256-274.

VAN NoORden, L. P. A. S. (1975). Temporal coherence in the perception of tone sequences. Unpublished doctoral dissertation, Eindhoven University of Technology.

VICARIO, G. (1973). Tempo Psicologico ed Eventi [Psychological time and events]. Florence: $\mathrm{C} / \mathrm{E}$ Giunti-G. Barbera.

VICARIO, G. (1982). Some observations in the auditory field. In J. Beck (Ed.), Organization and representation in perception (pp. 269-283). Hillsdale, NJ: Erlbaum.

\section{NOTES}

1. As a first approximation, independent sources can be thought of here as sources that can vary independently. From an organism's point of view, the independence of objects is probably a function of the different actions afforded the organism by one object versus another.

2. Perhaps it would be more precise to refer to this acoustic manipulation as " $F 1$-cutback" or "periodic-energy delay." However, the generally accepted articulatory term VOT will be used for ease of reading.

3. This difference in slope does not disappear with a transformation from frequency to mel.

4. Probit analysis is a class of nonlinear models of estimation for binary decision variables. For the current analyses, a cumulative normal was fitted to the identification function by transforming the percent of "bee" responses to $z$ scores and then finding the best fitting line through linear regression. The boundary was taken as the VOT value corresponding to $50 \%$ on this line.

5. Another possibility, pointed out by a reviewer, is that only lower frequency harmonics are relevant for stream segregation. This is in concert with the data on vowel sequence segregation from Dorman et al. (1975), where $F 1$ frequency was determinative of the perceptual groupings.

6. A shattering object may approximate an instantaneous change in shape, at least on the time scale of human perception. This results in a near instantaneous transient sound. Segregation processes are probably not important in this case.

(Manuscript received June 22, 1995; revision accepted for publication January 2, 1996.) 